\title{
LA INCORPORACIÓN DE LA REALIDAD AUMENTADA EN LAS CLASES DE CIENCIAS
}

\author{
Juan Carlos Rivadulla López \\ Universidad de A Coruña \\ Marisol Rodríguez Correa \\ Universidad Internacional de La Rioja
}

\begin{abstract}
RESUMEN: La Realidad Aumentada (RA) se considera una de las opciones de mayor proyección educativa gracias a su capacidad para conseguir mayor percepción, interacción y aprendizaje por parte del alumnado. El objetivo de este estudio es conocer y analizar las percepciones de un grupo de profesores de Primaria y Secundaria sobre la RA, las ventajas que otorga dicha tecnología y las necesidades para su incorporación. Han participado 806 profesores y se ha utilizado un cuestionario para la recogida de datos. Los resultados evidencian que la mayoría del profesorado indicó no haber implementado la RA en sus aulas. Los que la implementaron, señalan que aumenta de la motivación del alumnado y mejora su acción formativa. La mayoría nunca han asistido a cursos de formación para utilizar esta herramienta, considerando que es necesaria la misma, no solo en función de la tecnología sino con relación a los contenidos que se transmiten.
\end{abstract}

PALABRAS CLAVE: Nuevas tecnologías, Ciencias de la naturaleza, Enseñanza primaria, Enseñanza secundaria.

\section{INCORPORATION OF AUGMENTED REALITY IN SCIENCES CLASSROOM}

\footnotetext{
ABSTRACT: Augmented Reality (AR) is considered one of the options for greater educational projection thanks to its ability to achieve greater perception, interaction and learning on the part of students. The objective of this study is to know and analyze the perceptions of a group of Primary and Secondary teachers about RA, the advantages that this technology offers and the needs for its incorporation. 806 teachers participated and a questionnaire was used to collect data. The results show that the majority of the teaching staff indicated that they had not implemented AR in their classrooms. Those who implemented it, point out that it increases students' motivation and improves
} 
their formative action. Most have never attended training courses to use this tool, considering that it is necessary, not only in terms of technology but also in relation to the content that is transmitted.

KEYWORDS: New technologies, Natural sciences, Primary education, Secondary education.

Recibido: 06/11/2018

Aceptado: 10/06/2019

Correspondencia: Juan Carlos Rivadulla López, Universidad de A Coruña, Rúa da Maestranza 9, 15001 A Coruña. Email: juan.rivadulla@udc.es.

\section{INTRODUCCIÓN}

El uso de las Tecnologías de la Información de la Comunicación (TIC) constituye un importante factor de cambio para nuestras sociedades, contribuyendo estas tecnologías a la rápida obsolescencia de los conocimientos y a la emergencia de nuevos valores (Fernández, 2017). De la misma forma, las TIC ocupan actualmente un lugar significativo entre las prioridades educativas ya que las mismas contribuyen en el proceso de enseñanza-aprendizaje de los alumnos (Ariza y Quesada, 2014; Alderete, Di Meglio y Formichella, 2017; Mermoud, Ordoñez y Garcia Romano, 2017). Sin embargo, consideramos tal y como señala Méndez (2012), que el movimiento continuo que tiene el desarrollo de las tecnologías hace difícil establecer indicadores de reflexión que supongan señalar con precisión los cambios y ajustes que tendrían que hacerse a la educación para adaptarse a dicha movilidad.

Existen varios modelos pedagógicos que sostienen las teorías de aprendizaje de la nueva era digital. Autores como Wheeler (2012), plantean como teorías para aprender con tecnologías emergentes el constructivismo y la teoría de la complejidad, mientras que algunos autores aparecen con otros conceptos formulados tras el surgimiento de la web y el Internet, como el aprendizaje autorregulado (Beishuizen, Carneiro y Steffens, 2007), el conectivismo (Siemens, 2005) y el aprendizaje entre iguales (Corneli, 2012), entre otros. Algunas de estas pedagogías surgen alrededor de las tecnologías emergentes y su introducción en la educación, conformándose una nueva cultura del aprendizaje al ofrecer un potencial informacional, comunicativo, interactivo, colaborativo e innovador. Con este artículo se pretende acercar a una de esas denominadas tecnologías informáticas emergentes: la Realidad Aumentada.

\section{LA REALIDAD AUMENTADA Y SUS POSIBILIDADES EDUCATIVAS}

A partir de la importancia que ha ido adquiriendo la web 2.0, la reducción de costes de los equipos, y la fuerte penetración de los dispositivos móviles, entre otros, han emergido algunas tecnologías que sin duda alguna están siendo cada vez más impulsadas en nuestros tiempos. Una de estas tecnologías es la RA. Esta se caracteri- 
za por "la incorporación de información digital incluyendo imágenes, vídeo y audio en el mundo real, y pretende mezclar la realidad con lo virtual, lo que permite a los usuarios interactuar con los dos objetos, el físico y el digital" (Johnson, Becker, Cummins, Estrada, Freeman y Hall, 2016, p. 40). Cabero, García y Barroso (2016) por su parte, definen esta tecnología como la conjunción de información digital y física en tiempo real a través de diferentes dispositivos tecnológicos, para crear una nueva realidad, para lo cual tanto la realidad física como la digital son necesarias. Se crea de esta forma una escenografía mediática, que incrementa la calidad y cantidad de información a la cual el sujeto puede tener acceso.

La RA puede emplearse en una diversidad de campos tales como la publicidad, navegación, arte, marketing y ventas, entretenimiento y juegos, redes sociales, educación, entre otros (Kipper y Rampolla, 2013). Con relación al área educativa, la RA se presenta con fuertes posibilidades de aplicación en las Instituciones Educativas (De la Horra, 2017). Esta tecnología sin duda alguna, constituye un nuevo recurso en nuestras aulas que ofrece múltiples posibilidades y planteamientos novedosos, para ser implantado en diversas áreas del conocimiento. En este sentido, Prendes (2015), señala que la RA es una prometedora tecnología, ya presente en muchas aulas, que puede ayudar a mejorar el proceso de enseñanza-aprendizaje. No obstante, tal y como señalan Cabero, Fernández y Marín (2017) para que este pueda funcionar con validez, el estudiante debe encontrarse motivado para utilizarla durante el proceso formativo.

Las experiencias educativas sobre la implementación de la RA se han realizado en diferentes niveles educativos, desde Primaria (Fracchia, Alonso de Armiño y Martins, 2015), secundaria (Torres, García y Caballero, 2018), bachillerato (Merino, Pino, Meyer, Garrido y Gallardo, 2015), formación profesional (Llopis, 2013), hasta la Universidad (Garay, Tejada y Castaño, 2017), y en diferentes áreas curriculares. En el caso de las ciencias existen experiencias para aumentar las actitudes de los alumnos hacia la misma (Bressler y Bodzin, 2013); e investigaciones relacionadas con el área de Química (Merino et al., 2015) y de Física (De la Torre, Martin, Saorín, Carbonell y Contero, 2013).

Algunos estudios como los realizados por Kamarainen, Metcalf, Grotzer, Browne, Mazzuca, Tutwiler y Dede (2013) y Huang, Chen y Chou (2016), han arrojado que los alumnos que han tenido experiencias formativas con RA han mejorado sus resultados de aprendizaje y han aumenta su motivación y disfrute mientras aprenden. De la misma forma, investigaciones realizadas para medir el nivel de satisfacción de los alumnos en experiencias formativas con esta tecnología, han encontrado niveles altos de satisfacción y valoraciones muy positivas por parte del alumnado (Neven, Hala y Mohamed, 2011; De la Torre et al., 2013).

Por su parte, Rodríguez y Rivadulla (2017) realizaron un estudio cuyo objetivo fue conocer y analizar las percepciones de un grupo de profesores de la Facultad de Informática de la Universidad de A Coruña sobre el uso de la RA, las ventajas que proporciona esta herramienta en el aula y las necesidades para su incorporación. Los resultados del estudio arrojaron que aunque la mayoría del profesorado no ha implementado la RA en el aula, el mismo indicó que las ventajas de la incorporación de dicha herramienta serían el aumento de la motivación del alumnado, el desarrollo del aprendizaje en contextos reales, el poder percibir un objeto desde diferentes puntos de vista y la mejora de la acción formativa, entre otros. 
De la misma forma, Restrepo, Cuello y Contreras (2015), realizaron un estudio sobre juegos didácticos basados en RA como apoyo en la enseñanza de biología en estudiantes de básica primaria. Los resulados del estudio arrojaron que la RA como herramienta didáctica favorece el aprendizaje de las temáticas de la asignatura de biología, debido a que los estudiantes pueden aprender de manera interactiva y divertida, de tal manera que se logre captar su atención.

Por lo tanto, tal y como señalan Cabero y Barroso (2016), la RA facilita el desarrollo de una metodología constructivista de enseñanza-aprendizaje, ya que el alumno se convierte en una persona activa, el cual realiza sus propios descubrimientos, relaciona conocimientos previos, genera ideas y realiza experimentos. De esta forma, el alumno logra controlar el proceso de aprendizaje combinando lo real y lo virtual. Pero, aunque la utilización de la RA favorece el proceso de enseñanza-aprendizaje, debemos tener en cuenta que la tecnología por sí misma no produce cambios, por este motivo su éxito dependerá de los fines educativos, de la metodología y de las actividades empleadas (Cela, Esteve, Esteve, González y Gisbert, 2017; Fernández, 2017).

Por otra parte, con esta tecnología el docente puede crear contenidos digitales educativos propios y contextualizados, gracias a numerosas aplicaciones distribuidas de forma gratuita para su uso no comercial bajo la licencia pública general GNU como ARToolKit o Aumentaty Author que permiten crear, visualizar y manipular modelos 3D de forma rápida y sencilla. Asimismo, existen algunos repositorios de escenas de RA como el desarrollado por el Centro Aragonés de Tecnologías para la Educación (Cózar, De Moya, Hernández y Hernández, 2015).

De la misma forma, para la formación, esta herramienta presenta una serie de dificultades entre las cuales podemos mencionar las siguientes: falta de investigaciones, la novedad de la tecnología, la falta de objetos de aprendizaje para su incorporación a situaciones de enseñanza, la formación del docente para su incorporación educativa y la rapidez de su evolución (Cabero, 2017; Han, Jo, Hyum y So, 2015; Jamali, Fairuz, Wai y Oskam, 2015; Santos et al., 2016).

La mayoría de las investigaciones realizadas sobre la RA en ciencias se relacionan con experiencias para aumentar las actitudes de los alumnos hacia la misma. Sin embargo, no existen muchos estudios acerca de la valoración que da el profesorado sobre esta tecnología. El objetivo del presente estudio, es conocer y analizar las percepciones de un grupo de profesores de Primaria y Secundaria sobre la RA. Para ello, se parte de una pregunta de investigación que gira en torno a una única cuestión problema: ¿qué piensa un grupo de profesores de Educación Primaria y de Educación Secundaria Obligatoria (ESO) sobre la RA y cómo la utiliza en sus clases de Ciencias? A partir de esta pregunta se derivan una serie de objetivos generales y específicos. El general es conocer y analizar las percepciones de un grupo de profesores de Educación Primaria y ESO sobre la RA. Más concretamente, los objetivos específicos se centran en averiguar las ventajas que les aporta dicha tecnología al aplicarla en sus clases de Ciencias, conocer las necesidades para su incorporación, indagar sobre los recursos que el profesorado conoce y utiliza en sus aulas para implementar la RA y analizar diferencias estadísticas entre el profesorado de ambas etapas educativas. 


\section{Metodología}

\section{Población y muestra}

La población estuvo constituida por profesorado en activo que impartían clases de Ciencias en Educación Primaria y Secundaria en el noroeste de España (Galicia, Asturias y Castilla-León) durante el curso 2017-2018. El tamaño muestral definitivo ascendió a 806 profesores (478 mujeres -59,3\%- y 328 hombres -40,7\%-): 374 (46,4\%) impartían docencia en los últimos cursos de Primaria y 432 (53,6\%) impartían docencia en Secundaria. En cuanto a la situación profesional, todos ellos llevaban impartiendo docencia más de 10 años, lo cual nos aseguraba que los docentes tuvieron tiempo para realizar una formación continua, pudiendo realizar diferentes cursos para perfeccionar sus conocimientos.

\section{Instrumento}

Para dar respuesta a la intencionalidad exploratoria de este estudio, se ha seguido una metodología cuantitativa, un método no experimental y descriptivo (Cabezas y Casillas, 2014). Concretamente se utilizó un muestreo no probabilístico, accidental o incidental, condicionado por la disponibilidad de los sujetos a participar en el estudio (Martínez 2007). Este método utilizado de encuesta con vocación descriptiva tiene el objetivo de describir las características de la población a partir de un estudio detallado de las variables de interés (Torrado, 2004). Se empleó un cuestionario que nos permitió obtener una visión general del pensamiento docente sobre la RA. Los apartados que incluyó el cuestionario se elaboraron en torno a los objetivos marcados para esta investigación y, por supuesto, teniendo en cuenta toda la fundamentación teórica expuesta anteriormente.

Con el fin de validar el instrumento, se inició el proceso de validación de contenido a través de dos fases:

a) En primer lugar, se utilizó la técnica de jueces expertos. La primera versión del instrumento fue enviada a un grupo de 8 jueces expertos (Conejero, Claver, Fernández, González y Moreno, 2017) con las siguientes características: todos ellos eran profesores universitarios con posesión del título de doctor en Ciencias de la Educación y tenían experiencia en el campo de las nuevas tecnologías y de las Ciencias. Las variables objeto de estudio para la validez fueron: 1) Grado de pertinencia al objeto de estudio, 2) Grado de comprensión y adecuación de la redacción del ítem y 3) Consideración de si sobraba o faltaba algún ítem.

b) Los jueces expertos indicaron que se debía mejorar la redacción de algunos ítems para facilitar su comprensión y consideraron incluir preguntas relacionadas con la formación en esta temática, ya que el cuestionario inicial no las incluía. Se consideró oportuno tener en cuenta estas aportaciones, pues aportarían un enriquecimiento al contenido del trabajo. Teniendo en cuenta estas aportaciones, se aplicó el instrumento a una muestra piloto de 20 profesores (10 de Primaria y 10 de $\mathrm{ESO})$, con la finalidad de detectar problemas de funcionamiento de los ítems 
antes de su aplicación a la totalidad de la muestra (Expósito, Navarro, Thoilliez y López 2010), pero el resultado fue óptimo y no se necesitó realizar más cambios.

Finalmente, el cuestionario constó de 10 preguntas, de las cuales 6 iban dirigidas a todo el profesorado y 4 al que implementó en alguna ocasión la RA en sus aulas. De las cuestiones que iban dirigidas a todos, una pretende recoger información sobre el conocimiento de experiencias de la RA en las aulas (¿Conoces algunas experiencias o investigaciones sobre la $R A$ en distintos niveles educativos?), otra con relación a las ventajas de esta técnica (¿Cuáles consideras que son las ventajas de implementar esta tecnología en el aula?), otra pregunta relacionada con los elementos que son necesarios para dicha implementación (¿Cuáles consideras que son los elementos necesarios para implementar esta tecnología en el aula?), y la siguiente, sobre los recursos tecnológicos de los que dispone el docente (Para producir entornos de $R A$ se necesitan algunos elementos, ¿Podrías indicar cuáles se encontrarían a tu disposición como profesor para implementar dicha tecnología en el aula?). Por último, se plantean dos preguntas relacionadas con la formación en esta temática ( Has asistido a algún curso de formación para implementar la $R A$ en las aulas? y ¿Consideras necesaria la formación del profesorado para incorporar la $R A$ en el aula?). Cada una de las preguntas contenía una serie de ítems, de los cuales cada docente debía marcar los que considerase oportunos (en las preguntas ya se les indicaba si la respuesta era única o podía ser múltiple). Para analizar los datos obtenidos se halló el porcentaje del profesorado que marcó cada ítem en el cuestionario.

En cuanto a las 4 preguntas específicas que se plantean a los docentes que implementaron en alguna ocasión la RA en sus aulas, lo que se pretende es recoger información sobre dicha implementación ( Has implementado alguna vez la $R A$ como recurso en tu asignatura?, ¿Por qué usas la $R A$ en tu aula?, ¿Qué elementos usas para implementar la $R A$ en tu aula? y ¿Qué recursos tecnológicos usas al desarrollar proyectos de $R A$ en tu aula?).

\section{Procedimiento de recogida y análisis de datos}

La recogida de datos se realizó a principios del curso académico 2016/2017 a través de correo electrónico. Para ello, se envió un correo electrónico a distintos centros educativos del norte de España, solicitando consentimiento para enviar un cuestionario y que fuese distribuido entre el profesorado con el fin de que lo cubrieran y lo devolvieran de la misma forma que lo recibieron. La participación fue voluntaria y se garantizó el anonimato y la confidencialidad de los datos obtenidos. Para responderlo, se les concedió el tiempo que ellos consideraron oportuno, pero recordándoles que esta información era necesaria para poder avanzar en la investigación.

En un primer momento, el análisis de las respuestas recogidas en el cuestionario se analizó de forma directa, estableciéndose porcentajes, y fue realizado independientemente por dos investigadores, discutiendo las posibles discrepancias. Posteriormente, se realizó un análisis más detallado de las respuestas de un grupo reducido de profesores (94 en Primaria y 154 en ESO), los cuales indicaron que sí que trabajan la RA en sus aulas. Concretamente, se analizó el motivo por el cual esos profesores usan la RA, así como los elementos y recursos tecnológicos que emplean para ello. Además, se 
aplicó la prueba estadística de independencia de criterios Chi-cuadrado con 95\% de confianza para determinar la asociación entre las variables de interés (las distintas preguntas del cuestionario) y la categoría de etapas educativas (primaria y secundaria). Este análisis estadístico y su procesamiento se realizaron en el software SPSS versión 24.

\section{Resultados}

\section{a) Análisis de todo el profesorado (implementó y no implementó la RA en sus aulas)}

A modo general, en cuanto al conocimiento de experiencias educativas donde se haya trabajado con la RA, la mayoría de maestros de Primaria desconoce dichas experiencias. Los que sí conocen alguna experiencia indican mayoritariamente experiencias en la misma etapa educativa en la que ellos imparten clase. Por el contrario, el profesorado de ESO indica conocer experiencias, sobre todo en ESO, aunque cabe destacar que el 12,50\% muestra desconocimiento sobre experiencias relacionadas con la RA.

En relación a las ventajas de implementar la RA en las aulas, para la mayoría de los docentes de Primaria y ESO son que aumenta la motivación del alumnado y que mejora la acción formativa de los alumnos (figura 1). En menor medida, el profesorado de ambas etapas educativas también se refiere a la capacidad de los alumnos de interactuar con los objetos virtuales en una forma directa y natural mediante la manipulación de objetos reales, a la facilidad de la comprensión de fenómenos complejos ya que la RA permite al alumno descomponer un objeto o fenómeno en sus fases o etapas y a la destreza para percibir un objeto desde diferentes puntos de vista. Menos del $10 \%$ del profesorado señala como ventaja el que favorece el aprendizaje autónomo y colaborativo del alumnado. Por último, pocos son los profesores tanto de Primaria como de ESO que considera que esta tecnología no aporta beneficios en el aula.

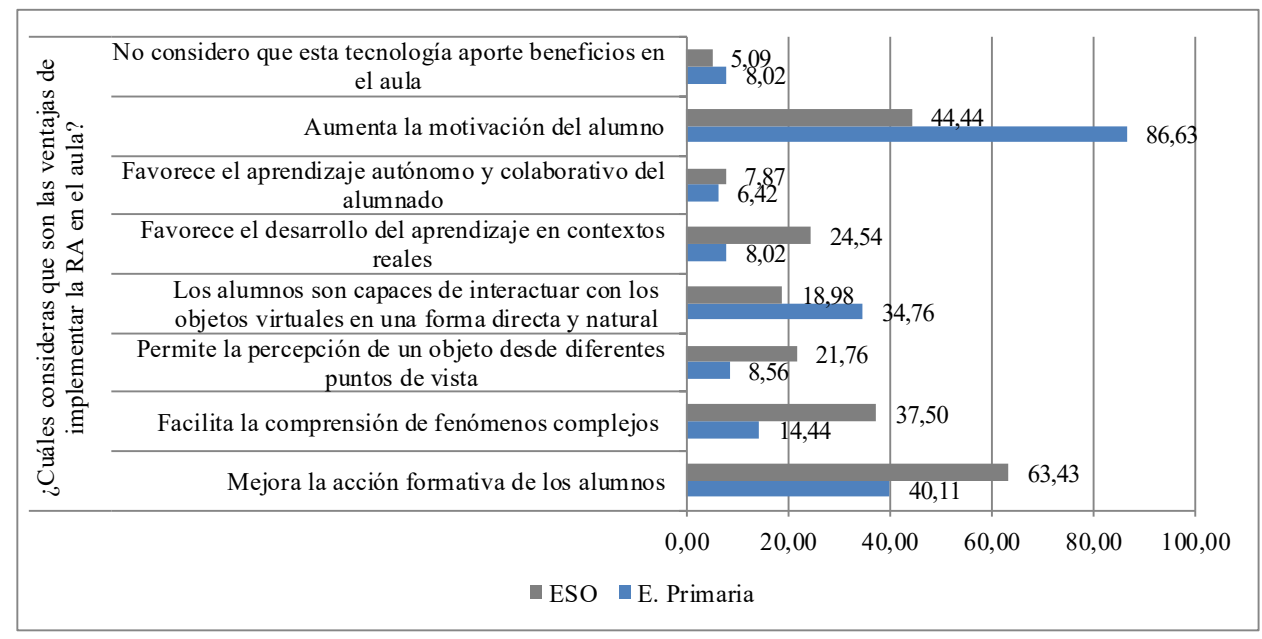

Figura 1. Respuestas del profesorado sobre las ventajas de implementar la $R A$ en el aula 
En relación a los recursos tecnológicos para desarrollar proyectos de RA en las aulas, el profesorado de Primaria y ESO indican desconocer dichos recursos (figura 2). En cuanto al profesorado que sí reconoce algunos de ellos, existe una tónica general y es que el profesorado de Secundaria tiende a conocer más recursos que los de Primaria. Concretamente, la mayoría hace referencia a Anatomia (trabaja en base a marcadores los contenidos esqueleto, cráneo, tronco y cuello y extremidades superiores e inferiores), Anatomy $4 d$ (permite el trabajo con los distintos sistemas del cuerpo humano, explorándolos en profundidad a través de la experiencia 4D, ofreciendo además la oportunidad de entender sus interrelaciones en el espacio), Aumentaty Autor (permite, por ejemplo, el trabajo con los sentidos (vista, oído, olfato, gusto), Corinth Anatomy (se puede elegir trabajar los diferentes sistemas del cuerpo) y Learn $A R$ (permite trabajar el cuerpo humano "órganos, corazón y algunos huesos"). En menor medida (menos del $20 \%$ del profesorado de ambas etapas educativas) se refieren a HeartCam (permite visualizar el corazón bajo rayos-x, en colores o un dibujo del mismo animado) e iSkull $A R$ (permite observar un cráneo muy realista, donde a través de colores se indican sus diferentes partes). Hay que destacar que el 5,56\% de los docentes de Secundaria y el 1,60\% de los de Primaria, proponen otros recursos a mayores de los que se les aportó en el cuestionario, entre ellos "librerias ARToolKit y BuildAr".

Respecto a los elementos que el profesorado tiene a su disposición para implementar la RA en sus aulas, el 40,11\% de los encuestados de Primaria y el $18,98 \%$ de ESO indican que no disponen de ninguno de esos recursos (figura 2). En cuanto al profesorado que sí reconoce que dispone de algunos de ellos, existe una tónica general y es que el de Secundaria tiende a disponer de más recursos que los de Primaria. Concretamente, la mayoría de los docentes de ESO cuentan con un elemento que captura la imagen de la realidad que están viendo los usuarios (pantalla del ordenador, un teléfono, o una videoconsola), un tipo de software específico para la producción del programa, un servidor de contenidos donde se ubica la información virtual que queremos incorporar a la realidad y un dispositivo donde proyectar la mezcla de las imágenes reales con las imágenes sintetizadas. Por su parte, el mayor porcentaje de maestros de Primaria que indica disponer de elementos para implementar la RA en sus aulas, se refiere a un tipo de software específico para la producción del programa y a un servidor de contenidos donde se ubica la información virtual que queremos incorporar a la realidad. En menor medida (menos del 15\% de los docentes de ambas etapas educativas), el profesorado de Secundaria cuenta con un elemento de procesamiento o varios que trabajen conjuntamente cuya función es la de interpretar la información del mundo real que recibe el usuario, generar la información virtual que cada servicio concreto necesite y mezclarla de forma adecuada (ordenadores, móviles o videoconsolas) y un activador de la RA o marcadores que pueden ser códigos QR, objetos físicos... Mientras, los maestros de Primaria, en menor medida cuenta con uno/varios elementos de procesamiento que trabajen conjuntamente, un activador de la RA y un dispositivo donde proyectar la mezcla de imágenes. 


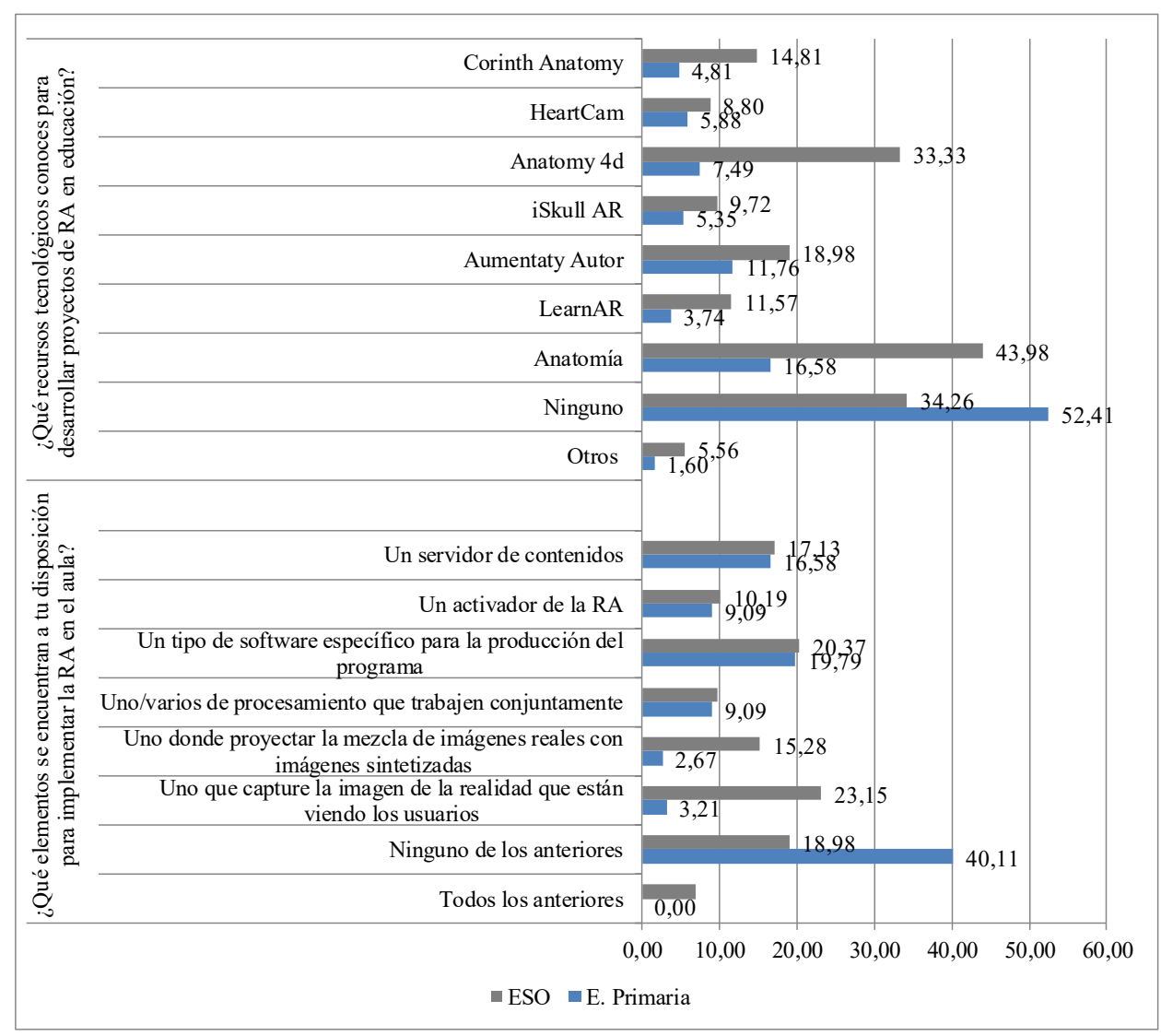

Figura 2. Respuestas del profesorado sobre los recursos tecnológicos que conoce para desarrollar proyectos de RA en educación y sobre los recursos de los que dispone

En relación a la formación docente, el 92,51\% de los participantes de Primaria y el $48,15 \%$ de Secundaria indica que nunca han asistido a cursos de formación y un $5,35 \%$ de Primaria y un $12,50 \%$ de Secundaria piensan que no se necesita formación pues con los conocimientos tecnológicos que poseen ya son suficientes (figura 3). No obstante, la mayoría del profesorado considera que sí es necesaria esa formación, y no solo en función de la tecnología sino en los contenidos que se transmiten. Una mayoría también considera es importante formación para incorporar la tecnología en el aula y piensan que es necesario contar con un buen asesoramiento de expertos, de una empresa desarrolladora de soluciones de RA o el apoyo de un grupo de investigación que comparte experiencias y recursos. 


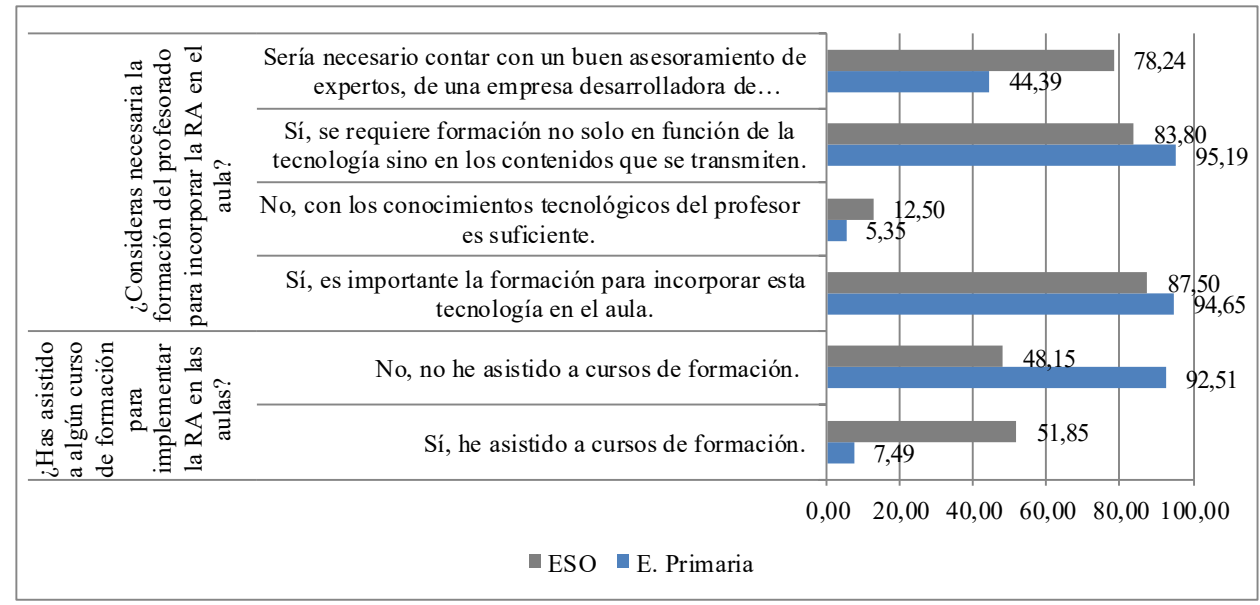

Figura 3. Respuestas del profesorado sobre las necesidades formativas en relación a la $R A$

\section{b) Profesorado que implementó la RA en sus aulas}

Por otro lado, en cuanto al profesorado que implementó alguna vez la RA en sus aulas, en Primaria solo indicó haberlo hecho el 25,13\% mientras que en Secundaria lo hizo el $35,65 \%$. A continuación, se exponen los resultados de las tres preguntas concretas que se les hizo a los profesores que indicaron que sí habían implementado alguna vez la RA en sus aulas. Así, en relación a las ventajas que encontraron al implementar la RA en su aula, se pueden encontrar diferencias entre el profesorado de ambas etapas educativas, aunque también alguna similitud. La mayoría de los docentes, tanto de Primaria como de Secundaria, indica que usa la RA en su aula para aumentar la motivación del alumnado, porque mejora la acción formativa de los alumnos y porque sirve al alumnado para interactuar con los objetos virtuales en una forma directa y natural mediante la manipulación de objetos reales (figura 4). Entre otras ventajas señaladas sobre esta herramienta, aunque con un porcentaje superior en secundaria que en primaria, se encuentran las siguientes: la RA facilita la comprensión de fenómenos complejos, permite la percepción de un objeto desde diferentes puntos de vista favorece el aprendizaje autónomo y colaborativo del alumnado y el aprendizaje en contextos reales.

Respecto a los elementos que el profesorado usa al implementar la RA en sus aulas, excepto cuando se habla de un tipo de software específico para la producción del programa, donde los maestros de Primaria indican usarlo más que el de Secundaria, generalmente el profesorado de Secundaria tiende a usar más recursos que los de Primaria (figura 5). Así, la mayoría de los docentes de Secundaria señalan utilizar un servidor de contenidos, mientras que el de Primaria, aunque también valora alto este ítem, lo hace en menor porcentaje. El profesorado en general valora en mucha menor medida otros elementos que usa en sus aulas, 
ya sea un activador de la RA o marcadores que pueden ser códigos QR, objetos físicos... o uno/varios elementos de procesamiento que trabajen conjuntamente (ordenadores, móviles o videoconsolas). Por último, cabe destacar que existen dos elementos que solo el profesorado de Secundaria señala usarlos: uno donde proyectar la mezcla de imágenes reales con imágenes sintetizadas y uno que captura la imagen de la realidad que están viendo los usuarios (pantalla del ordenador, un teléfono, o una videoconsola).

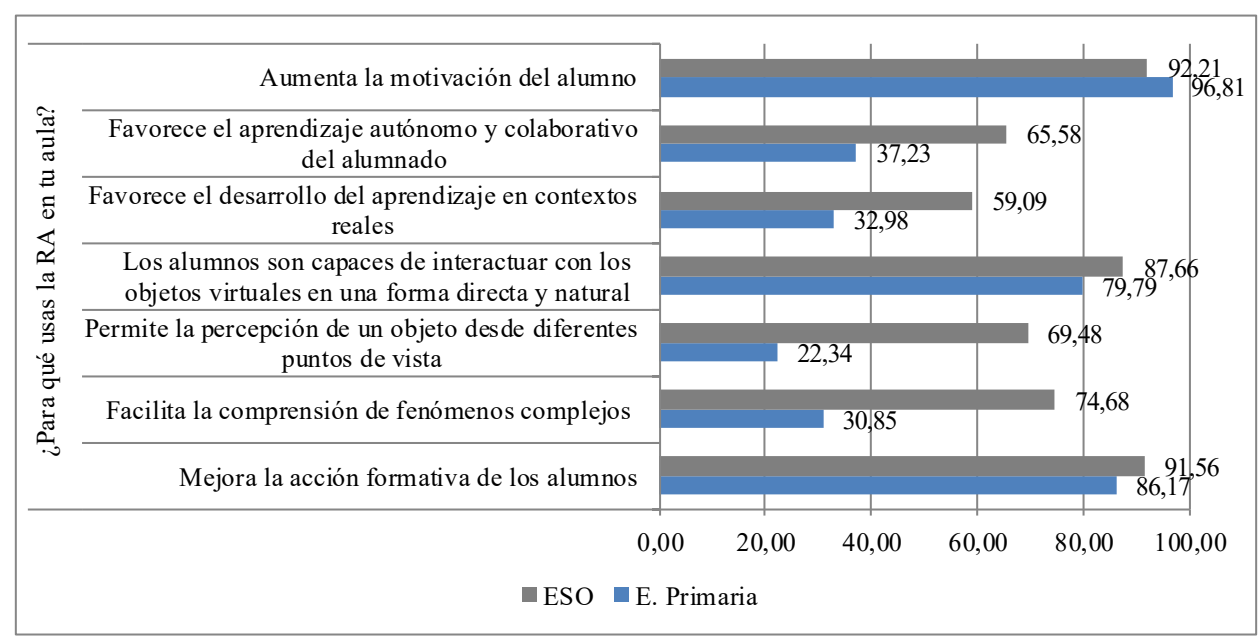

Figura 4. Respuestas del profesorado sobre las ventajas que encontraron al implementar la RA en su aula

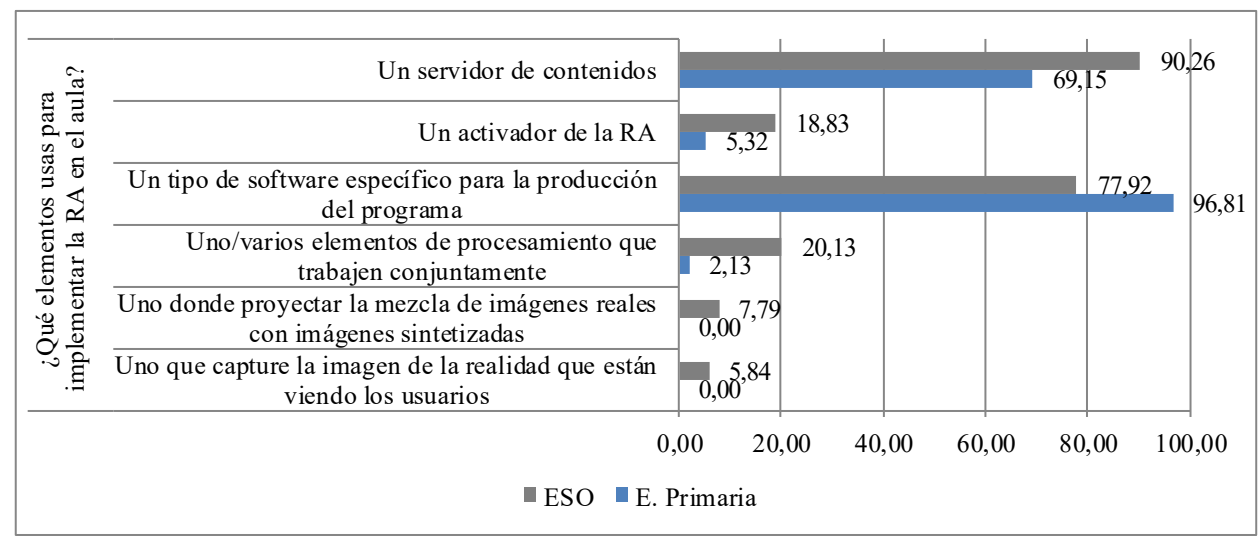

Figura 5. Respuestas del profesorado sobre los elementos que usa para desarrollar proyectos de $R A$ en sus aulas 
En relación a los recursos tecnológicos empleados para desarrollar proyectos de RA en las aulas, los docentes de Secundaria tienden a utilizar en mayor medida más recursos que los de Primaria (figura 6). Concretamente, la mayoría hace referencia a Anatomia, Anatomy 4d, Corinth Anatomy y Aumentaty Autor. En menor medida (menos del 30\% del profesorado de ambas etapas educativas) se refieren a Learn $A R$, iSkull $A R$ y HeartCam. Cabe destacar que el 5,84\% de los docentes de Secundaria y el $2,13 \%$ de los de Primaria, emplean otros recursos a mayores de los que se les aportó en el cuestionario (librerias ARToolKit y BuildAr).

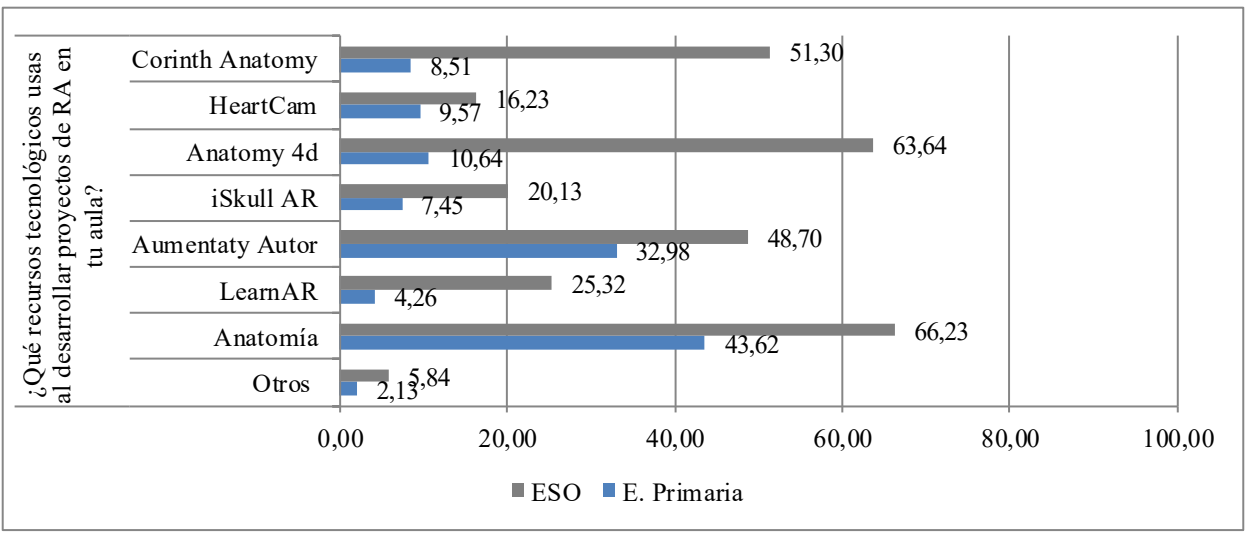

Figura 6. Respuestas del profesorado sobre los recursos tecnológicos que usa para desarrollar proyectos de RA en sus aulas

\section{c) Análisis individualizado de los participantes}

Este análisis muestra un perfil diferenciado entre el profesorado de Primaria y de Secundaria (tabla 1). Aunque la mayoría del profesorado de ambas etapas educativas dice no usar la RA en sus aulas y entre ellos no existen evidencias significativas $(p=0,769)$, si existen tales diferencias en cuanto a la necesidad formativa que muestran los docentes $(p=0,001)$ y la asistencia a cursos sobre RA $(p=0,000)$. En cuanto al número de ventajas que le otorgan al uso de la RA en las aulas, al número de recursos que conocen y al número de elementos que tienen a su disposición, la mayoría del profesorado de ambas etapas indican entre 1 y 4 para las dos primeras categorías y entre 1 y 3 para la última; no obstante, existen diferencias significativas entre estas categorías y la etapa educativa $(p=0,000 ; p=0,009$ y $p=0,021$ respectivamente).

En relación al profesorado que indicó haber implementado en sus aulas la RA en alguna ocasión, las diferencias entre etapas educativas son más notables. El número de ventajas que le otorgan a la RA los maestros de Primaria oscila mayoritariamente entre 3 y 5, mientras que en Secundaria entre 6 y 7. Lo mismo ocurre con el número de elementos y de recursos que utilizan, pues en Primaria oscila entre 1 y 2 en ambos 
casos, y en Secundaria entre 3 y 6 y entre 3 y 4 respectivamente. Los resultados obtenidos con la prueba de Chi Cuadrado de Pearson $\left(\chi^{2}\right)$ en el número de ventajas del uso de la RA ( $p=0,005)$, el número de elementos que usa el profesorado $(p=0,019)$ y el número de recursos utilizados $(p=0,011)$, muestran evidencias significativas para rechazar la hipótesis nula, destacando que estas categorías están relacionadas con la etapa educativa.

Tabla 1. Relación entre la etapa educativa del profesorado y las diferentes categorías incluidas en el cuestionario (frecuencia y Chi Cuadrado de Pearson $\left(\chi^{2}, p<0,05\right)$ ).

\begin{tabular}{|c|c|c|c|c|c|c|}
\hline \multicolumn{7}{|c|}{ TODOS LOS PARTICIPANTES } \\
\hline \multicolumn{2}{|l|}{$\begin{array}{l}\text { Categorías } \\
F\end{array}$} & \multicolumn{2}{|c|}{$\begin{array}{c}\text { Educación } \\
\text { Primaria } \\
(\mathbf{n}=\mathbf{3 7 4})\end{array}$} & \multicolumn{2}{|c|}{$\begin{array}{c}\text { Educación } \\
\text { Secundaria } \\
(\mathbf{n = 4 3 2})\end{array}$} & \multirow[t]{2}{*}{$\chi^{2}$} \\
\hline & & $\%$ & $F$ & $\%$ & & \\
\hline \multirow{2}{*}{ Uso de la RA } & Sí & 94 & 25,1 & 154 & 35,6 & \multirow{2}{*}{0,769} \\
\hline & No & 280 & 74,9 & 278 & 64,4 & \\
\hline \multirow{3}{*}{$\begin{array}{l}N^{\circ} \text { de ventajas del uso de } \\
\text { la RA }\end{array}$} & $5-7$ & 19 & 5,1 & 59 & 13,7 & \multirow{3}{*}{0,000} \\
\hline & $1-4$ & 325 & 86,9 & 351 & 81,2 & \\
\hline & Ninguna & 30 & 8 & 22 & 5,1 & \\
\hline \multirow{3}{*}{$N^{o}$ de recursos que reconoce } & $5-8$ & 19 & 5,1 & 58 & 13,4 & \multirow{3}{*}{0,009} \\
\hline & $1-4$ & 139 & 37,2 & 190 & 44 & \\
\hline & Ninguno & 216 & 57,7 & 184 & 42,6 & \\
\hline \multirow{3}{*}{$\begin{array}{l}N^{0} \text { de elementos a su } \\
\text { disposición }\end{array}$} & $4-6$ & 21 & 5,6 & 130 & 30,1 & \multirow{3}{*}{0,021} \\
\hline & $1-3$ & 203 & 54,3 & 220 & 50,9 & \\
\hline & Ninguno & 150 & 40,1 & 82 & 19 & \\
\hline \multirow{2}{*}{ Necesidad Formativa } & Sí & 354 & 94,7 & 378 & 87,5 & \multirow{2}{*}{0,001} \\
\hline & No & 20 & 5,3 & 54 & 12,5 & \\
\hline \multirow{2}{*}{ Asistencia a cursos } & Sí & 28 & 7,5 & 198 & 45,8 & \multirow{2}{*}{0,000} \\
\hline & No & 346 & 92,5 & 234 & 54,2 & \\
\hline \multicolumn{7}{|c|}{ PROFESORADO QUE IMPLENTÓ LA RA EN SUS AULAS } \\
\hline \multirow{2}{*}{\multicolumn{2}{|c|}{$\begin{array}{l}\text { Categorías } \\
F\end{array}$}} & \multicolumn{2}{|c|}{$\begin{array}{c}\text { Educación } \\
\text { Primaria } \\
(\mathrm{n}=94) \\
\end{array}$} & \multicolumn{2}{|c|}{$\begin{array}{c}\text { Educación } \\
\text { Secundaria } \\
(\mathrm{n}=154)\end{array}$} & \multirow[t]{2}{*}{$\chi^{2}$} \\
\hline & & $\%$ & $F$ & $\%$ & & \\
\hline \multirow{3}{*}{$\begin{array}{l}N^{\circ} \text { de ventajas del uso de } \\
\text { la RA }\end{array}$} & $6-7$ & 28 & 29,8 & 101 & 65,6 & \\
\hline & $3-5$ & 46 & 48,9 & 53 & 34,4 & 0,005 \\
\hline & $1-2$ & 20 & 21,3 & 0 & 0 & \\
\hline \multirow{3}{*}{$\mathrm{N}^{0}$ de elementos que usan } & $5-6$ & 0 & 0 & 9 & 5,8 & \\
\hline & $3-4$ & 5 & 5,3 & 138 & 89,6 & 0,019 \\
\hline & $1-2$ & 89 & 94,7 & 7 & 4,6 & \\
\hline \multirow{3}{*}{$\mathrm{N}^{\circ}$ de recursos que usan } & $7-8$ & 5 & 5,3 & 33 & 21,4 & \\
\hline & $3-6$ & 13 & 13,8 & 72 & 46,8 & 0,011 \\
\hline & $1-2$ & 76 & 80,9 & 49 & 31,8 & \\
\hline
\end{tabular}




\section{Discusión}

Con nuestro trabajo de investigación hemos pretendido conocer y analizar las percepciones de un grupo de profesores de Primaria y Secundaria sobre la RA, determinando las ventajas que otorga dicha tecnología y las necesidades para su incorporación.

Los resultados del estudio aportan evidencia empírica con relación a algunas diferencias halladas entre lo expresado por los profesores participantes de las etapas de Primaria y Secundaria, como son las siguientes:

- La mayoría del profesorado de Primaria desconoce experiencias educativas que hayan empleado la RA, y los que conocen alguna experiencia señalaron que es en su misma etapa educativa. Por el contrario, la mayoría del profesorado consultado en ESO señaló conocer experiencias donde se ha implementado esta herramienta.

- El profesorado de Primaria consultado en nuestro estudio indicó que la principal ventaja de implementar la RA en el aula es que aumenta la motivación del alumnado, seguido de que mejora la acción formativa de los alumnos. Por otra parte, la mayoría del profesorado de Secundaria señaló como ventaja más significativa la mejora de la acción formativa de los alumnos y en segundo lugar el aumento de la motivación del alumnado.

- En relación a los recursos tecnológicos para desarrollar proyectos de RA en las aulas, más de la mitad de los maesrtos de primaria los desconoce, al contrario que el profesorado de Secundaria, quiénes en su mayoría señalaron conocer más recursos. Algunos de los recursos tecnológicos mencionados por un porcentaje significativo de los docentes consultados para producir entornos de RA son: Anatomía y Anatomy 4d, Aumentaty Autor y Corith Anatomy. Otros de los recursos identificados en la encuesta realizada, aunque en menor medida fueron Heartcam e iSkull AR.

- Respecto a los elementos que el profesorado tiene a su disposición para implementar la RA en sus aulas, la mayoría de los docentes consultados reconocieron que disponen de algunos de ellos, aunque los docentes de Secundaria disponen de más recursos que los de Primaria, entre ellos: elementos que capturan la imagen de la realidad que están viendo los usuarios (pantalla del ordenador, un teléfono, o una videoconsola), softwares específico para la producción de programas, servidores de contenidos donde se ubica la información virtual para incorporar a la realidad y dispositivos donde proyectar la mezcla de las imágenes reales con las imágenes sintetizadas.

\section{Conclusiones}

Las evidencias derivadas de este estudio descriptivo sirven a las pretensiones de mejora y desarrollo competencial de los estudiantes, así como a las del profesorado. De los resultados obtenidos, podemos extraer una serie de conclusiones que nos permiten situarnos con mayor conocimiento ante el papel desempeñan las nuevas 
tecnologías en la educación, en específico la RA en el aula y la actitud del profesorado respecto a la misma:

- La mayoría del profesorado de Primaria como de Secundaria indicó no haber implementado la RA en sus aulas, aspecto que concuerda con las investigaciones de Gómez, Cañas, Gutiérrez y Martín (2014) y de Garófalo, Chemes y Alonso (2016), las cuales señalan que muchos docentes de distintos niveles educativos tienen dificultades para incorporar las TIC a sus secuencias didácticas. No obstante, se puede destacar que dentro del grupo de docentes que señaló que sí la ha implementado, los Secundaria han utilizado más esta herramienta.

- Con relación a las ventajas que señalaron el grupo de profesores que, si han implementado la RA en su aula, la mayoría coincide en que esta herramienta aumenta la motivación del alumnado, mejora la acción formativa de los alumnos y sirve al alumnado para interactuar con los objetos virtuales en una forma directa y natural mediante la manipulación de objetos reales, Estos resultados coinciden con los estudios realizados por Kamarainen et al. (2013), Huang et al. (2016) y Fracchia et al. (2015), según los cuales, la RA genera un gran interés en el alumnado, complementando los materiales didácticos existentes con modelos virtuales que estimulan la percepción y ayudan a la comprensión de los conceptos.

- Entre los elementos que han utilizado para emplear la RA, la mayoría del profesorado utiliza diferentes tipos de software que son específicos para la producción de programas y servidores de contenidos, y en menor medida activadores de la RA o marcadores que pueden ser códigos QR u objetos físicos, y elementos de procesamiento que trabajen conjuntamente (ordenadores, móviles o videoconsolas). Así mismo, los docentes de Secundaria utilizaron en mayor medida más recursos que los maestros de Primaria para poder implementar la RA en el aula, entre los que se pueden destacar: Anatomia, Anatomy $4 d$, Corinth Anatomy y Aumentaty Autor. Algunos docentes proponen herramientas a mayores de las que aportó el cuestionario como las librerías ARToolkit y BuildAR. Consideramos tal y como señala Buenaventura (2014), que existen en el mercado una amplia gama de herramientas informáticas y tecnológicas que permiten crear una aplicación de RA, ya sean de licencia de uso gratuito o de pago. Los docentes deberán elegir cuál herramienta utilizar dependiendo del contexto en donde se utilizará la aplicación. Por lo tanto, antes de utilizar un Programa previamente se deben evaluar los objetivos y alcance del sistema de software, para así optimizar el uso de los recursos disponibles.

- En relación a la formación docente, la mayoría del profesorado consultado de ambas etapas educativas indicaron que nunca han asistido a cursos de formación. De la misma manera, indicaron que sí es necesaria esa formación, no solo en función de la tecnología sino con relación a los contenidos que se transmiten, además de un asesoramiento por parte de expertos o el apoyo de un grupo de investigación que comparta experiencias y recursos. Estos resultados coinciden por lo expuesto por Vaden (2013), cuando señala que es 
importante que el profesorado durante todo el desarrollo de los proyectos con RA, continúe realizando búsquedas que les permitan "nutrirse" con nueva información. Por lo tanto, no sólo es fundamental la investigación con respecto a la utilización de los programas a trabajar, sino también con respecto al tema elegido y a los contenidos.

- Consideramos tal y como señalan Cela et al. (2017), que la competencia digital formará parte del conjunto de requerimientos de un perfil profesional, en este caso del ejercicio de la profesión de docente, por lo que será relevante realizar planteamientos relacionados con el desarrollo curricular, con las estrategias didácticas y con todos aquellos aspectos que permitan diseñar acciones formativas orientadas a que el docente utilice la tecnología de forma didáctica con sus estudiantes.

\section{REFERENCIAS BIBLIOGRÁFICAS}

Alderete, V., Di Meglio, G. y Formichella, M. (2017). Acceso a las TIC y rendimiento educativo: ¿una relación potenciada por su uso? Un análisis para España. Revista de Educación, 377, 54-81. http://doi.org/10.4438/1988-592X-RE-2017-377-353.

Ariza, M. R. y Quesada, A. (2014). Nuevas tecnologías y aprendizaje significativo de las ciencias. Enseñanza de las Ciencias, 32(1), 101-115.

Beishuizen, J., Carneiro, R. y Steffens, K. (EDS.) (2007). Self-regulated Learning in Technology Enhanced Learning Environments: Individual Learning and Communities of Learners. Proceedings of the KALEIDOSCOPE-TACONET Conference. Aachen: Shaker Verlag.

Bressler, D. M. y Bodzin, A. M. (2013). A mixed methods assessment of students' flow experiences during a mobile augmented reality science game. Journal of Computer Assisted Learning, 29(6), 505-517. http://doi.org/10.1111/jcal.12008.

Cabero, J. y Barroso, J. (2016). Posibilidades educativas de la Realidad Aumentada. New Approaches In Educational Research, 5(1), 46-52. http://doi.org/10.7821/ naer.2016.1.140

Cabero, J., García, F. y Barroso, J. (2016). La producción de objetos de aprendizaje en "Realidad Aumentada": Ia experiencia del SAV de la Universidad de Sevilla. International Journal of Educational Research and Innovation (IJERI), 6, 110-123.

Cabero, J. (2017). Presentación: Aplicaciones de la Realidad Aumentada en Educación. Revista de Educación Mediática y TIC, 6 (1), 4-8.

Cabero Almenara, J., Fernández Robles, B. y Marín Díaz, V. (2017). Dispositivos móviles y realidad aumentada en el aprendizaje del alumnado universitario. RIED. Revista Iberoamericana de Educación a Distancia, 20(2).

Cabezas, M. y Casillas, S. (2014). Percepción de los alumnos de Educación Primaria de la Universidad de Salamanca sobre su competencia digital. EDUTEC, Revista Electrónica de Tecnología Educativa, 48, 1-14. 
Cela, J., Esteve, V., Esteve, F. M., González, J. y Gisbert, M. (2015). El docente en la sociedad digital: una propuesta basada en la pedagogía transformativa y en la tecnología avanzada. Profesorado. Revista de Currículum y Formación del Profesorado, 21(1), 403-422.

Conejero, M., Claver, F., Fernández, C., González, J. y Moreno, M.P. (2017). Diseño y validación de un instrumento de observación en voleibol. CCD, 34(13), 67-75.

Corneli, J. (2012). Paragogical Praxis. E-Learning and Digital Media, 9(3), 267-272.

Cózar, R., De Moya, M. V., Hernández, J. A. y Hernández, J. R. (2015). Tecnologías emergentes para la enseñanza de las Ciencias Sociales. Una experiencia con el uso de Realidad Aumentada en la formación inicial de maestros. Digital Education Review, 27, 138-153.

De la Horra, G. I. (2017). Realidad Aumentada, una revolución educativa. Edmetic, Revista de Educación Mediática y TIC, 6(1), 9-22.

De la Torre, J., Martín, N., Saorín, J. L., Carbonel, C. y Contero, M. (2013). Entorno de aprendizaje ubicuo con realidad aumentada y tabletas para estimular la comprensión del espacio tridimensional. RED, Revista de Educación a Distancia, 37. Recuperado de http://www.um.es/ead/red/37.

Expósito, E., Navarro, E., Thoilliez, B. y López, E. (2010). Determinants of child well-being: A perspective from students of education. Comunicación presentada en la European Conference on Educational Research (ECER), Helsinki, Finlandia.

Fernández, B. (2017). Factores que influyen en el uso y aceptación de objetos de aprendizaje de realidad aumentada en estudios universitarios de Educación Primaria. Edmetic, Revista de Educación Mediática y TIC, 6(1), 203-220.

Fracchia, C., Alonso de Armiño, A. C. y Martins, A. (2015). Realidad Aumentada aplicada a la enseñanza de Ciencias Naturales. TE Y ET. Realidad Aumentada aplicada a la enseñanza de Ciencias Naturales, 16, 7-15.

Garay, U., Tejada, E. y Castaño, C (2017). Percepciones del alumnado hacia el aprendizaje mediante objetos educativos enriquecidos con realidad aumentada. Edmetic, Revista de Educación Mediática y TIC, 6(1), 145-164.

Garófalo, S. J., Chemes, L. B. y Alonso, M. (2016) Propuesta didáctica de enseñanza con simulaciones para estudiantes del profesorado en Ciencias Biológicas. Revista Eureka sobre Enseñanza y Divulgación de las Ciencias, 13(2), 359-372.

Gómez M. A., Cañas A. M., Gutiérrez M. S. y Martín M. J. (2014). Ordenadores en el aula: ¿estamos preparados los profesores? Enseñanza de las ciencias, 32 (2), 239-250.

Han, J., Jo, M., Hyun, E. y So, H. (2015). Examining young children's perception toward augmented reality-infused dramatic play. Education Technology Research Development, 63, 455-474. 
Huang, T.C., Chen, C.C. y Chou, Y.W. (2016). Animating eco-education: To see, feel, and discover in an augmented reality-based experiential learning environment. Computers \& Education, 96, 72-82. http://doi.org/10.1016/j.compedu.2016.02.008.

Jamali, S., Fairuz, M., Wai, K. y Oskam, Ch. (2015). Utilising mobile-augmented reality for learning human anatomy. Procedia-Social and Behavioral Sciences, 197, 659-668. http://doi. org/10.1016/j.sbspro.2015.07.054.

Johnson, L., Becker, S., Cummins, M., Estrada, V., Freeman, A. y Hall, C. (2016). NMC Horizon Report: 2016. Higher Education Edition. Austin, Texas: The New Media Consortium.

Kamarainen, A., Metcalf, S.H., Grotzer, T., Browne, A., Mazzuca, D., Tutwiler, M. y Dede, Ch. (2013). EcoMOBILE: Integrating augmented reality and probe-ware with environmental education field trips. Computers and Education, 68, 545-556. http://doi.org/10.1016/j.compedu.2013.02.018.

Kippler, G. y Rampolla, J. (2013). Augmented reality. Amsterdam: Syngress.

Llopis, B (2013). Realidad aumentada. Revista Didáctica, Innovación y Multimedia, 26.

Martínez, R. A. (2007). La investigación en la práctica educativa: Guía metodológica de investigación para el diagnóstico y la evaluación en los centros docentes. Madrid: Ministerio de Educación y Ciencia.

Merino, C., Pino, S., Meyer, E., Garrido, J. y Gallardo, F. (2015). Realidad aumentada para el diseño de secuencias de enseñanza-aprendizaje en química. Educación química, 26(2), 94-99. http://doi.org/10.1016/j.eq.2015.04.004.

Mermoud, S. R., Ordoñez, C. y García Romano, L. (2017) Potencialidades de un entorno virtual de aprendizaje para argumentar en clases de ciencias en la escuela secundaria. Revista Eureka sobre Enseñanza y Divulgación de las Ciencias 14(3), 587-600.

Neven A. M., Hala, H. y Mohamed, I. (2011). ARSC: Augmented reality student card. An augmented reality solution for the educational field. Computers and education, 56, 1045-1061. http://doi.org/10.1016/j.compedu.2010.10.019.

Prendes, C. (2015). Realidad Aumentada y Educación: análisis de experiencias prácticas. Píxel-Bit. Revista de Medios y Educación, 46, 187-203. http://doi. org/10.12795/pixelbit.2015.i46.12.

Restrepo, D., Cuello, L. y Contreras, L. (2016). Juegos didácticos basados en realidad aumentada como apoyo en la enseñanza de la Biología. Ingeniare, 11(19), 99116.

Rodríguez, M. y Rivadulla, J. (2017). Uso y ventajas de la realidad aumentada en el aula. International Conference on Innovation, Documentation and Education. Innodoct 2017, 439-448. 
Santos, M., Wolde, A., Taketomi, T., Yamamoto, G., Rodrigo, M., Sandor, CH. y Kato, H. (2016). Augmented reality as multimedia: the case for situated vocabulary learning. Research and Practice in Techology Enhanced Learning, 11(4), 1-23.

Siemens, G. (2005). Connectivism: A learning theory for the digital age. International Journal of Instructional Technology and Distance Learning, 2 (1), 3-10. Recuperado de http://www.itdl.org/Journal/Jan_05/article01.htm.

Torrado, M. (2004). Estudios de encuesta. En R. Bisquerra. Metodología de la investigación educativa (231-257). Barcelona: La Muralla.

Torres, I., García, E. y Caballero, M. (2018). La Realidad Aumentada y las Ciencias Experimentales: diseño de actividades para el aula de Educación Secundaria. Comunicación presentada en 28 Encuentros de Didáctica de las Ciencias Experimentales, A Coruña, España.

Vadén, L. (2013). Aprendiendo biología con realidad virtual y realidad aumentada. Dirección Sectorial de Planificación Educativa. Sembrando experiencias. Recuperado el 13 de enero de 2018 de http://www.anep.edu.uy/sembrando/index. php/ciencias-naturales/40-aprendiendo-biologia-con-realidad-virtual-y-realidadaumentada.

Wheeler, S. (2012). Theories for the digital age. Recuperado el 25 de noviembre de 2017 de http://stevewheeler.blogspot.com.es. 